\title{
Prevalencia de erosión dental en escolares de Tampico, Madero, Altamira y su relación con el pH salival
}

Gutiérrez D

Isassi $H$

Oliver $R$

Padilla J

Trejo E

Huitzil E

\section{Resumen}

Introducción: La erosión dental es la pérdida de la superficie del diente causada por el ataque ácido y es un problema de salud dental en el mundo moderno; determinar su etiología y factores de riesgo permitirán tratarla y prevenirla correctamente antes de que se presente un daño más severo. Objetivo: Identificar la prevalencia de erosión dental en escolares de Tampico, Madero, Altamira y su relación con el pH salival. Material y Métodos: Fue un estudio observacional y transversal, se examinaron a 510 escolares de Tampico, Madero y Altamira. Se utilizó el índice de erosión de Aine. Resultados: De los 510 escolares, 63 presentaron una frecuencia de erosión dental del $12.35 \%$, de los cuales 35 $(13.67 \%)$ fueron niñas y $28(11.02 \%)$ niños. No se encontraron diferencias estadísticamente significativas entre niños y niñas $(\mathrm{p}=.36)$. En relación con el pH salival no se encontró diferencias estadísticamente significativas. $(\mathrm{p}=.11)$. El órgano dental más afectado fue el $46(7.20 \%)$ y el 63 $(4.93 \%)$. El grado que más prevaleció fue grado $1(8.03 \%)$. En cuanto a la superficie más afectada fue la vestibular. Conclusiones: Es importante para el odontopediatra detectar los signos de erosión en etapas iníciales, identificar la causa y vigilar la nutrición de los niños que presentan erosión para prevenirla oportunamente.

Palabras claves: Erosión, pH, saliva, riesgo.

Diana Monserrat-Gutiérrez-Oviedo. Alumna Postgrado de Odontopediatría Facultad Odontología Universidad Autónoma de Tamaulipas. Autor responsable.

M.C.D.E.O: Hilda-Isassi-Hernández Coordinador Postgrado Odontopediatría Facultad Odontología Universidad Autónoma de Tamaulipas México M.C.D.E.E: Rogelio-Oliver-Parra. Asesor de clínica del posgrado de Endodoncia Facultad Odontología Universidad Autónoma de Tamaulipas México

M.C.D.M.E.S: Juventino-Padilla-Corona. Maestro Titular Materia Odontología Preventiva Facultad Odontología Universidad Autónoma de Tamaulipas México

M.C.D.E.O: Sergio E.-Trejo-Tejeda. Asesor Clínica Postgrado Odontopediatría Facultad Odontología Universidad Autónoma de Tamaulipas México M.D.U: Enrique E.-Huitzil-Muñoz. Profesor Titular, Maestría Ciencias Estomatológicas, Benemérita Universidad Autónoma de Puebla, México 


\section{Prevalência de erosão dentária em escolares Tampico, Madero, Altamira e sua relação com pH salivar}

\section{Resumo}

Introdução: A erosão dentária é a perda da estrutura do dente causada pelo ataque ácido e é um problema de saúde bucal no mundo moderno; determinar sua etiologia e fatores de risco permitirá tratá-la e preveni-la corretamente antes que se desenvolvam danos mais severos. Objetivo: Identificar a prevalência de erosão dentária em escolares de Tampico, Madero, Altamira e sua relação com o pH salivar. Material e Métodos: Através de estudo observacional e transversal, foram examinados 510 escolares de Tampico, Madero e Altamira. Utilizou-se o índice de erosão de Aine. Resultados: Dos 510 escolares, $63(12,35 \%)$ apresentaram erosão dentária, sen- do 35 meninas $(13,67 \%)$ e $28(11,02 \%)$ meninos. Não se encontraram diferenças estatisticamente significantes entre os gêneros $(p=0,36)$. Em relação ao $\mathrm{pH}$ salivar não se encontraram diferenças estatisticamente significativas. $(p=0,11)$. Os elementos dentais mais afetados foram o 4.6 $(7,20 \%)$ e o $6.3(4,93 \%)$. O grau que mais prevaleceu foi o $1(8,03 \%)$ e a superfície vestibular foi a mais afetada. Conclusões: É importante para o odontopediatra detectar os sinais de erosão em etapas iniciais, identificar a causa e vigiar a alimentação das crianças que apresentam erosão para preveni-la oportunamente.

Palavras chave: Erosão, dental, $\mathrm{pH}$, saliva, risco.

\section{Prevalence of dental erosion in school children of Tampico Madero Altamira and its relation to the salivary $\mathrm{pH}$}

\begin{abstract}
Introduction: Dental erosion is the loss of tooth surface caused by acid attack and is a dental health problem in the modern world, determining its etiology and risk factors will allow properly treating and preventing it before the damage done is more severe. Objective: To identify the prevalence of dental erosion in schoolchildren in Tampico, Madero, Altamira and its relation to salivary $\mathrm{pH}$. Material and Methods:
\end{abstract}

It was a cross-sectional observational study, 510 schoolchildren were examined in Tampico, Madero and Altamira. We used the Aine index for dental erosion. Results: Out of the 510 schoolchildren, 63 had a frequency of dental erosion of $12.35 \%$, of which $35(13.67 \%)$ were girls and $28(11.02 \%)$ boys. There were no statistically significant differences between boys and girls ( $p$ $=.36$ ). In relation to salivary $\mathrm{pH}$, no statistically significant differences were found. $(P=.11)$. The 
most frequently affected dental organs were 46 $(7.20 \%)$ and $63(4.93 \%)$. The most prevalent grade was grade1 $(8.03 \%)$. In regards to the area, the most affected was the vestibular. Conclusions: It is important for the pediatric dentist to detect signs of erosion in its early stages, identify the cause and monitor the nutrition of children with erosion to treat and prevent this condition.

Key words: Dental, erosion, salivary, ph, risk.

\section{Introducción}

Imfeld T. en 1996 describió los procesos destructivos crónicos que afectan a los dientes además de la caries dental, los cuales producen una pérdida irreversible de la estructura dentaria, encontrándose entre ellos la abrasión, atrición, abfracción y la erosión. ${ }^{1,2,5,6,14,16,19,22} \mathrm{La}$ erosión dental se deriva del latín erodere, erosi, y erosum (corroer), y se define como la pérdida progresiva e irreversible de tejido dental por un proceso químico que no involucra la acción bacteriana. ${ }^{1,2,3}$ Lazarchik, concluyó que la erosión comienza con la desmineralización superficial del esmalte, provocando la disolución de las capas del diente y la eventual pérdida de estructura dental dejándolos frágiles y sensibles al dolor. ${ }^{4}$ Asher (1987), Habsha (1999), Ibbetson (1999), Lazarchik (2000), Al-Majed I. y cols., (2002) entre otros, clasifican los factores de erosión dental en intrínsecos y extrínsecos. En los intrínsecos encontramos la bulimia, rumia o fenómeno de reflujo voluntario, insuficiencia subclínica debido a la gastritis crónica asociada con el alcoholismo, xerostomía, síndrome de mala absorción, vómitos crónicos durante el embarazo y ERGE. Los extrínsecos incluyen gaseosas, bebidas, alimentos ácidos, pastillas cítricas, algunos medicamentos, sustitutos de saliva, exposición al agua de las piscinas con cloro y la exposición ocupacional a los agentes corrosivos, (vapores de ácidos de las baterías y gases de industrias). 4,5,6,7.14,15,23,24,26,27 Los índices utilizados en prevalencia de erosión dental son: el índice de erosión dental de Smith y Knight, índice de Eccle's, índice de Aine, índice de Larsen y Westergaar y el índice de O’Sullivan. 8,9,10, 18, 19, 21,22

Nuñez (1990) y Leone y cols., (2001) mencionaron que en la cavidad oral existen grandes protectores del medio, dentro de los cuales destaca la saliva, sin embargo, la saliva presenta amplias variabilidades en su comportamiento. ${ }^{11,12} \mathrm{El} \mathrm{pH}$ salival puede ser afectado por diversos factores como el tipo de dieta, ingesta de agua etc. demostrando con ello una alta variabilidad en las condiciones salivales entre distintos sujetos y en un mismo sujeto durante el día. ${ }^{11,12,13,17,18,20,28,29}$

Skjorland y cols. (1995), Hannig (1999) mencionaron en su estudio que la saliva es responsable de la formación de película adquirida, que es una barrera física que protege el diente contra ataques erosivos, la cual está compuesta de una capa de proteínas formada sobre la superficie del diente, actuando como una barrera de difusión o como una membrana permeable. ${ }^{13,14}$ En cuanto a la prevención de la erosión dental, Imfeld (1996) sugiere utilizar cepillos de cerdas suaves para la higiene bucal con pastas dentales no abrasivas y con fluoruros, no cepillar inmediatamente después del vómito, postergar una hora el cepillado para posibilitar la acción reparadora de los iones salivales Ca y P., estimular el flujo salival con gomas de mascar con xilitol $u$ otros sustitutos de la sacarosa y control odontológico para aplicación de fluoruros con $\mathrm{pH}$ neutro cada 3 meses en los casos de gravedad clínica. ${ }^{1,9,25}$

El objetivo del estudio fue identificar la prevalencia de erosión dental en escolares de Tampico, Madero y Altamira Tamaulipas y su relación con el pH salival. 


\section{Material y métodos}

Se realizó un estudio observacional y transversal, considerando como universo a los escolares de Tampico, Madero y Altamira, Tamaulipas en el período de Diciembre del 2009 a Noviembre del 2010.

- Etapa I.- Calibración.

- Etapa II.- Platica informativa a padres de familia y firma de consentimiento.

- Etapa III.-Procedimiento: se examinaron a 510 escolares a luz natural, se utilizó un espejo plano \#4 TBS con mango recto y explorador TBS, guantes y cubrebocas. Los datos obtenidos de los escolares que presentaron erosión dental fueron registrados en el formato recolector de erosión dental de acuerdo a la clasificación de Aine (1993) que agrupa a dientes temporales como permanentes.

\section{Tabla 1.}

En la presente investigación se manejó frecuencia y porcentaje para las variables cualitativas y para las variables numéricas, medias y desviación estándar. Las pruebas estadísticas empleadas fueron Chi-Cuadrado para la comparación de variables cualitativas, y la prueba $\mathrm{T}$ de Student para grupos independientes en el caso de las variables cuantitativas; las pruebas se manejaron a un valor alfa .05 en el programa estadístico Stat View versión 5.0 (Abacus Concepts).

\section{Resultados}

La muestra total fue de 510 escolares, 256 (50.19\%) sexo femenino y $254(49.80 \%)$ masculino. De los 510 escolares revisados, 63 presentaron erosión dental (12.35\%). Figuras $\mathbf{1}$ y 2.

De los 63 escolares (12.35\%) con erosión, 35 fueron niñas $(13.67 \%)$ y 28 niños $(11.02 \%)$. En la relación de erosión dental y el $\mathrm{pH}$ salival no se encontraron diferencias estadísticamente significativas $(\mathrm{p}=.11)$. El porcentaje de las piezas permanentes y temporales más afectadas por la erosión dental se observa en la figura $\mathbf{3}$ y 4 .

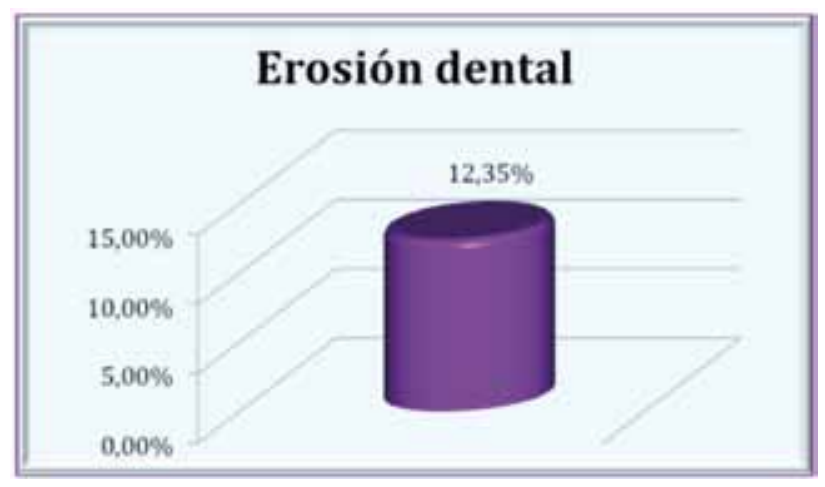

Figura 1. Escolares que presentaron erosión dental.

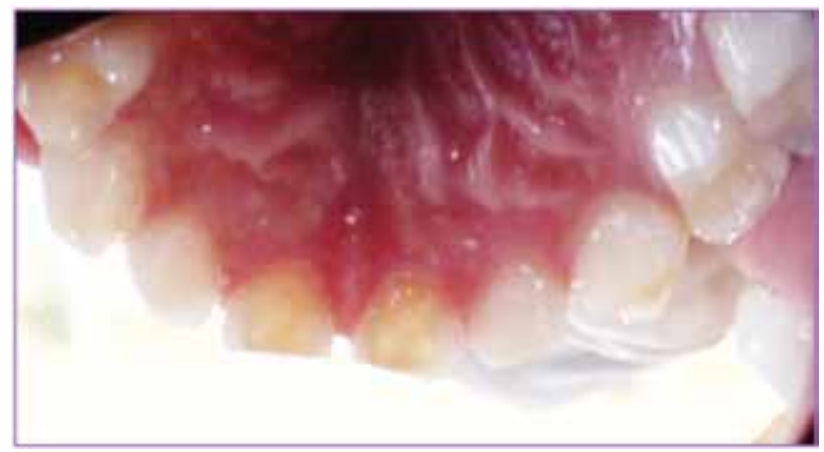

Figura 2. Erosión dental.

Tabla 1. Clasificación de erosión dental Aine 1993

\begin{tabular}{|c|c|}
\hline Grado 0 & No existe erosión dental. \\
\hline Grado 1 & Aparición de opacidades o manchas blancas en los dientes. \\
\hline Grado 2 & Superficies oclusales con pequeñas cavidades. \\
\hline Grado 3 & $\begin{array}{r}\text { Exposición de dentina en el fondo de las cavidades en las superficies oclusales o } \\
\text { dentina afectada en otras superficies. }\end{array}$ \\
\hline
\end{tabular}




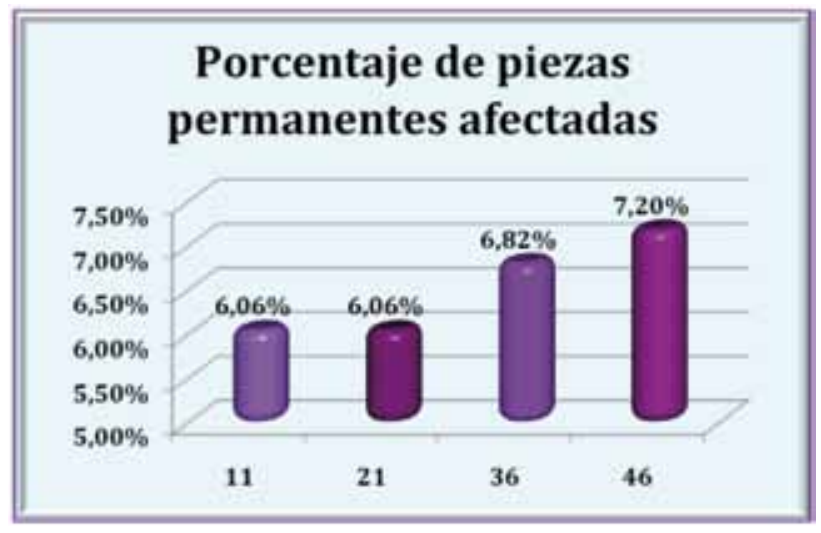

Figura 3. Porcentaje de piezas permanentes más afectadas por erosión dental.

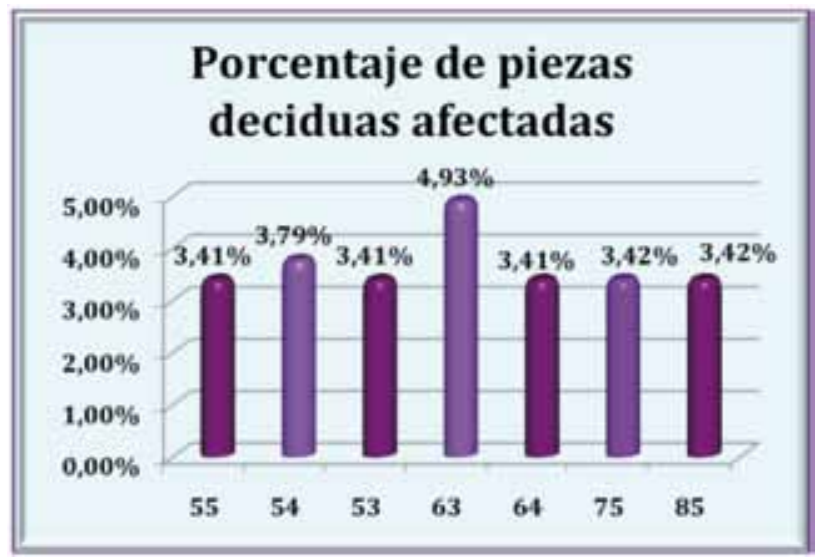

Figura 4. Porcentaje de piezas temporales más afectadas por la erosión dental.

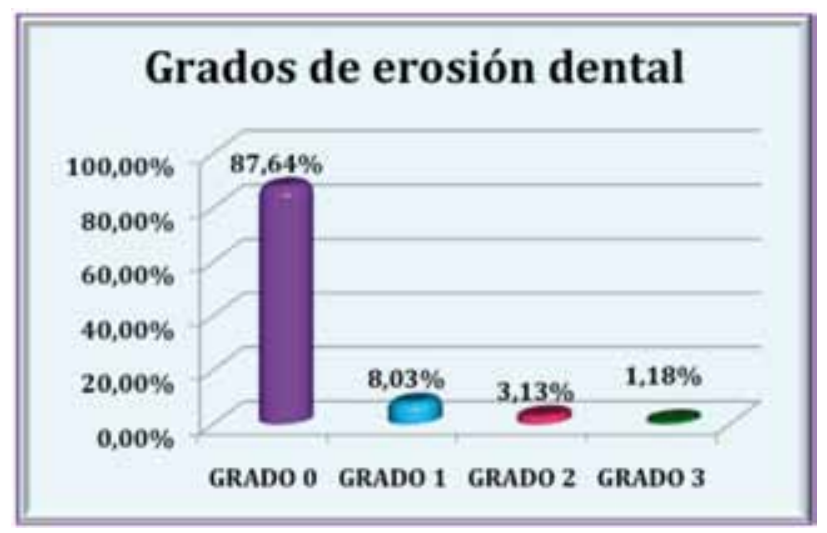

Figura 5. Porcentaje de grado de erosión dental que más prevaleció.

El grado de erosión dental que más prevaleció en los 510 escolares se muestra en la Figura 5.

En el grado de erosión por género no se identificaron diferencias estadísticamente significativas $(\mathrm{p}=.67)$.

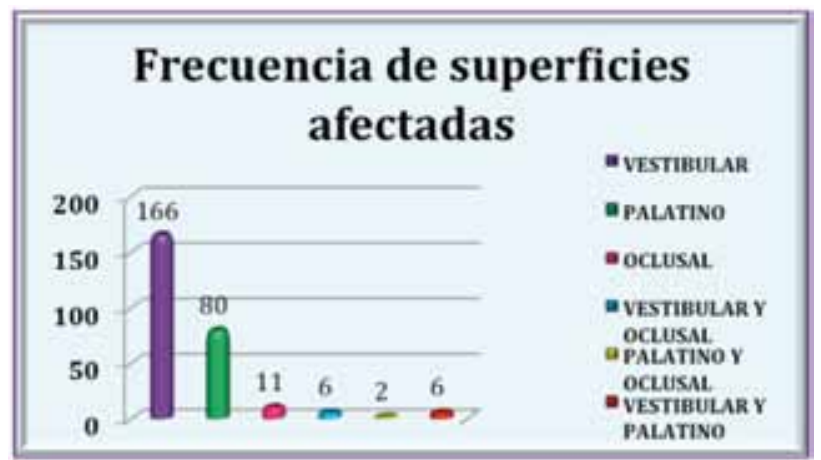

Figura 6. Frecuencia de las superficies del diente más afectados por la erosión dental.

En cuanto a frecuencia de las superficies del diente más afectados por la erosión dental se muestra en la figura 6 y la frecuencia de superficie mas afectada por género se observa en la tabla 2.

No se encontró diferencias estadísticamente significativas en las superficies afectadas por género $(\mathrm{p}=.0015)$.

Tabla 2. Porcentaje de superficies dentales afectadas por género.

\begin{tabular}{|c|c|c|}
\hline \multirow{2}{*}{ SUPERFICIE } & MASCULINO & FEMENINO \\
\cline { 2 - 3 } & $\mathbf{n} \%$ & $\mathbf{n} \%$ \\
\hline VESTIBULAR & $76(45.78 \%)$ & $90(54.21 \%)$ \\
\hline PALATINO & $51(63.75 \%)$ & $29(36.25 \%)$ \\
\hline OCLUSAL & $7(63.63 \%)$ & $4(36.36 \%)$ \\
\hline $\begin{array}{c}\text { VESTIBULAR Y } \\
\text { OCLUSAL }\end{array}$ & $0(0.00 \%)$ & $6(100 \%)$ \\
\hline $\begin{array}{c}\text { PALATINOY } \\
\text { OCLUSAL }\end{array}$ & $1(50.00 \%)$ & $1(50.00 \%)$ \\
\hline $\begin{array}{c}\text { VESTIBULAR Y } \\
\text { PALATINO }\end{array}$ & $6(100 \%)$ & $0(0.00 \%)$ \\
\hline
\end{tabular}




\section{Discusión}

Es importante conocer y detectar los signos de erosión en etapas iniciales (sensibilidad, decoloración, transparencia etc.) antes de que ocurra un daño más severo. El porcentaje de erosión dental encontrada fue de $12.35 \%$, más bajo que en la mayoría de los resultados reportados por otros autores, sin embargo resultados similares fueron observados por Peres y cols. (2005) quienes en 499 niños encontraron 13\% de erosión dental en escolares. Por el contrario Al-Majed y cols. (2002), en adolescentes de 12 a 14 años de edad, encontró erosión dental en un 26\%. Deery y cols. (2000) observó una prevalencia muy alta de erosión dental en los EE.UU. (41\%) entre los niños de 11 a 13 años. ${ }^{16,17,18}$

El estudio realizado por Al-Dlaigan y cols. (2001) en Birmingham, Reino Unido, encontró una prevalencia de $48 \%$ en una población de 14 años de edad. Posiblemente la diferencia de los resultados se debe a la presencia de otros factores en la aparición de erosión dental, por ejemplo, la variabilidad en hábitos y dieta entre poblaciones pueden influir con la presencia de una mayor erosión ${ }^{19}$.

En nuestro estudio no se encontraron diferencias estadísticamente significativas en la prevalencia de erosión dental entre niños y niñas, muy similar al estudio realizado por Peres y cols. (2005) y Deery y cols. (2000). Por lo que se deduce que la participación de la erosión se da por igual en ambos géneros según lo reflejan estos resultados. ${ }^{16,18}$

Es necesario enfatizar que la presencia de erosión se debe a diversos factores, determinar su etiología permitirá al odontopediatra poder tratar y prevenirla correctamente; en esta investigación centramos la atención en la relación que la erosión puede guardar con el pH salival, sin embargo como lo reportamos, los escolares con y sin erosión presentaron un $\mathrm{pH}$ similar. Lussi. (2000), West. (1998), Hunter. (2000), Grace. (2004) y Hay. (1962) reportaron el carácter multifactorial que guarda la erosión dental. 21, 22, 23, 24,25

Estamos de acuerdo que existen numerosos factores de riesgo por los cuales puede descender el $\mathrm{pH}$ a nivel crítico y que no sólo es responsabilidad de la saliva, si no que se asocia a diferentes factores, como lo reportaron autores, como AlMajed y cols. (2002), Berner y cols. (2004), Cebrián y cols. (2006), Cabrera y cols. (2004) entre otros. Mencionaron que el valor de $\mathrm{pH}$ es una variable significativa en el proceso de erosión, pero no es necesariamente el factor más importante. De los 510 escolares examinados, no se encontraron diferencias estadísticamente significativas en la relación de erosión dental y el pH salival con el género. ${ }^{17,26,27,28}$

Künzel y cols. $(2000)^{20}$ en su estudio de erosión asociado al consumo excesivo de naranjas, reportó que el grado 1 predominó en un $13.3 \%$. De igual forma observamos que el grado 1 fue el que prevaleció en nuestra investigación. Con ello observamos que la erosión presentada en la población estudiada no solamente es baja, sino que en los casos que se presentó fue la más incipiente. También encontramos una frecuencia y porcentaje de $54 \%$ de erosión dental en la superficie vestibular de niñas, seguido por la superficie palatina $(63 \%)$ en niños. Estos resultados son coincidentes con los encontrados por Peres y cols. (2005) ${ }^{16}$ donde encontró que la superficie vestibular y palatina fueron las más afectadas encontrando un $31.3 \%$ y $24.7 \%$ de erosión respectivamente. La presencia de reflujo gastroesofágico o bulimia puede ser responsable de la erosión en la superficie palatina en el caso de los niños, más no es clara por que esta situación no apareció en las niñas. El predominio de la erosión en 
la superficie vestibular obtenido en este estudio, también coincide con los estudios de Williams y cols. (1999) y Al-Majed y cols. (2002) ${ }^{17,29}$. Estos resultados pueden llevar a asociar a que algunos de los factores extrínsecos predominan en esta población más que algún factor intrínseco aunque es solo una hipótesis ya que en nuestro estudio no se realizó alguna encuesta para conocer factores de riesgo.

\section{Conclusiones}

Bajo las condiciones y limitaciones del estudio establecemos las siguientes conclusiones:
1. La presencia de erosión dental fue de $12.35 \%$ y no se encontró relación con un $\mathrm{pH}$ salival bajo.

2. El grado de erosión más observado fue el grado 1, y los dientes afectados con mayor frecuencia fueron los molares permanentes inferiores y de la dentición temporal el canino superior izquierdo.

3. La erosión dental en escolares de sexo masculino se presentó más en la superficie palatina y en el sexo femenino fue la superficie vestibular.

\section{Referencias}

1. Imfeld T. Dental erosion. Definición, classification and links. Eur J Oral Sci. 1996; 104(2) (Pt2):151-155.

2. Flores F NS, Gil O NC, San Martin B W, Hernández T NG, Galindo M J. Prevalencia de erosión dental en niños con diagnóstico de enfermedad por reflujo gastroesofágico en el Hospital Para El Niño Poblano. Rev. Acad. Mex. Odon. Ped. 2009: 21(2):46-49.

3. Eccles JD. Tooth surface loss from abrasion, attrition and erosion. Dent Update. 1982: 9: 373-374, 376-378, 380-381.

4. Lazarchik DA, Filler SJ. Dental erosion: Predominant oral lesion in gastro esophageal reflux disease. Am J Gastroenterology 2000; 958 Suppl:S33-8.

5. Asher C, Read MJ. Early enamel erosion in children associated with the excessive consumption of citric acid. Br Dent J 1987; 162(10):384-7.

6. Habsha E. The etiology and pathogenesis of tooth wear: Part I. Oral Health 1999; 83-92.

7. Ibbetson R, Eder A. Tooth Surface loss: editors' introduction. Br Dent J 1999; 186 (2); 60-6.

8. Berg BG, Kutschmann M, Bardehle D. Methodological considerations concerning the development of oral dental erosion indexes. Literature survey, validity and reliability. Clin Oral Invest .2008; 12: S51-S58.

9. Engelen L, De Wijik RA, Prinz JF, Van der Bilt A, Bosman F. The relation between saliva flow after different stimulations and the perception of flavor and texture attributes in custard dessert. Physiol Behav. 2003; 78: 165-169.

10. Servari R, Meurman JH, Alakuijala P, FrankRM. Effect of fluoride varnish and solution on enamel erosion in vitro. Caries Res. 1994; 28 227-232.

11. Núñez G. Estudo do metebolismo de carboidratos em núcleos de células de glándulas salivares durante o desenvolvimento da sialose. Universidad de Sao Paulo, 1990, Brazil.

12. Leone C, Oppenheim F. Physical and chemical aspects of saliva as indicators of risk for dental caries in humans. J Dent Educ. 2001; 65: 1054-62.

13. Hannig M. Ultrastructural investigation of pellicle morphogenesis at two different intraoral sites during a 24 -h period. Clin Oral Inves. 1999; 3: 88-95.

14. Skjorland KK, Rykke M, Sonju T. Rate of pellicle formation in vivo. Acta Odontol Scand. 1995; 53: 358-362.

15. Imfeld T. Prevention of progression of dental erosion by professional and individual prophylactic measures. Eur $\mathrm{j}$ oral Sci 1996; 104:215-220. 
16. Peres KG, Armeño MF, Peres MA, Traebert J, De Lacerda JT. Dental erosión in 12 year-old schoolchildren: a crosssectional study in Southern Brazil. International Journal of Pediatric Dentistry. 2005; 15: 249-255.

17. Al-Majed I, Maguirre A, Murray JJ. Prevalence and risk factors for dental erosion in 5-6 year old and 12-14 year old boys in Saudi Arabia. Community Dent Oral Epidemiol. 2002; 30: 38-46.

18. Deery C, Wagner ML, Longbotton C, Simon A, Nugent ZJ. The prevalence of dental erosion in a United States and a United Kingdom sample of adolescents. Pediatric Dentistry 2000; 22: 505-510.

19. Al-Dlaigan YH, Shaw L, Smith A. Dental erosion in a group of British 14-year-old school children. Part I. Prevalence and influence of differing socioeconomic backgrounds. British Dental Journal 2001; 190: 145-149.

20. Künzel W, Cruz MS, Fischer T. Dental erosion in Cuban children associated with excessive consumption of oranges. Eur J Oral Sci 2000; 108: 104-109.

21. Lussi A, Kohler N, Zero D, Schaffner M, Megert B. A comparison of the erosive potential of different beverages in primary and permanent teeth using an in vitro model. Eur J Oral Sci. 2000; 108(2):110-4.

22. West NX, Maxwell A, Huges JA, Parker DM, Newcombe RG, Addy M. A metodh to measure clinical erosion: Deffect of orange juice consumption on erosion of enamel. J Dent 1998; 26: 329-35.

23. Hunter ML, West NX, Hughes JA, Newcombe RG. Addy M. Relative susceptibility of deciduos and permanent dental hard tissues to erosion by allow pH fruit drink in vitro. J Dent 2000B; 28: 265-70.

24. Grace EG, Sarlani E, Kaplan S. Tooth erosión caused by chewing aspirin. J Am Dent Assec 2004; 135:191-4.

25. Hay DL, Pinset BR, Schram CJ, Wagg BJ. The protective effect of calcium and phosphate ions against acidic erosion of dental enamel and dentine. Br Dent J 1962; 3:283-7.

26. Berner E, Piñero J, Valente S. Trastornos de la conducta alimentaria. Signos clínicos en pacientes adolescentes con episodios de vómitos autoinducidos. 2004; 102(6):440-444.

27. Cebrian CJL, Lopez- Arca CJM. Diagnóstico de reflujo gastro-esofágico mediante impregnación por ácido de férula oclusal. Med Oral Patol Oral Cir Bucal 2006; 11:E26-8.

28. Cabrera AR, Kanashiro CR. Efecto del pH del agua de piscina en esmalte de dientes deciduos humanos. Estudio con microscopía electrónica de barrido. Rev Estomatol Herediana 2004; 14 (1-2).

29. Willians D, Croucher R, Marcenes W, O'Farrel M. The prevalence of dental erosion in the maxillary incisors of 14-yearold schoolchildren living in Tower Hamlets and Hackney, London, UK. International Dental Journal 1999; 49: 211-216.

Recibido para evaluación: 25-05-11

Envío evaluación: 26-05-11

Aceptado: 12-08-11

Correspondencia: hisassi@uat.edu.mx 